\title{
Principles of Discrete Time Mechanics: IV : The Dirac equation, particles and oscillons
}

\author{
Keith Norton and George Jaroszkiewicz \\ Department of Mathematics, University of Nottingham \\ University Park, Nottingham NG7 2RD, UK
}

June 20, 2018

\begin{abstract}
We apply the principles of discrete time mechanics discussed in earlier papers to the first and second quantised Dirac equation. We use the Schwinger action principle to find the anticommutation relations of the Dirac field and of the particle creation operators in the theory. We find new solutions to the discrete time Dirac equation, referred to as oscillons on account of their extraordinary behaviour. Their principal characteristic is that they oscillate with a period twice that of the fundamental time interval $T$ of our theory. Although these solutions can be associated with definite charge, linear momentum and spin, such objects should not be observable as particles in the continuous time limit. We find that for non-zero $T$ they correspond to states with negative squared norm in Hilbert space. However they are an integral part of the discrete time Dirac field and should play a role in particle interactions analogous to the role of longitudinal photons in conventional quantum electrodynamics.
\end{abstract}

\section{Introduction}

Throughout this paper we shall use the acronyms $C T$ to denote continuous time and $D T$ to denote discrete time. The symbol $T$ is used to denote our fundamental interval of time, which is assumed positive. The term CT limit and the symbol $\underset{T}{\rightarrow}$ will refer to the taking of $T$ to zero and some integer $n$ to infinity, such that $n T \underset{T}{\rightarrow} t$, where $t$ is ordinary continuous co-ordinate time, assuming that this limit makes sense. This will frequently be the case, but it should always be kept in mind that $D T$ mechanics is inherently richer in its range of possibilities than $C T$ mechanics and includes trajectories for which such a limit is meaningless. This is particularly the case for the oscillon solutions which we shall discuss in the case of the DT Dirac equation. 
This paper is the fourth in a series devoted to the construction of $D T$ classical and quantum mechanics from first principles. This series of papers examines two questions: first, whether it is possible or consistent to rewrite all the laws of physics using discrete time rather than continuous time, and second, if such a programme were viable and self consistent, what would be the advantages (and perhaps disadvantages) of such a discrete time formulation. Throughout this series we invert the standard view of discrete time. Rather than regarding it as a convenient approximation to continuous time, suitable for say computer simulation, we suppose from the outset that our ordinary conception of time is itself an approximation, albeit an extraordinarily useful one, and that deep down there is a scale at which temporal phenomena occur in a discrete way and not in a differentiable or even continuous way. An analogy with water is useful here. For a vast range of phenomena, water may be sensibly modelled as a continuum, but sooner or later a molecular approach must be used.

Our philosophy imposes a rigid discipline on our work. Our principles of discrete time mechanics must be as well specified as those of continuous time mechanics. Our laws of motion must be exact, and our invariants of the motion cannot be approximately conserved but must be exactly conserved modulo those equations of motion. We should avoid introducing ad-hoc fixes for problems when they occur, but rely solely on the basic starting assumptions. In this respect we have found that our basic principles have guided us well, so that once a calculation has been started, we have found virtually no freedom of choice thereafter. What has emerged is a consistent theory of mechanics, both classical and quantum, which is genuinely different from continuous time mechanics, but which has sufficient overlap with it in so many ways as to make further investigation highly desirable.

A question of some delicacy for $D T$ mechanics is the issue of Lorentz covariance. Our DT mechanics is not manifestly Lorentz covariant. Fortunately, it turns out from our studies of scalar and Dirac field propagators that Lorentz covariance and the Poincaré algebra appear to be broken in $D T$ mechanics only at the order $T^{2}$ level so that our field theoretic scattering amplitudes are expected to have an expansion in powers of $T$ such that the zeroth order terms are Lorentz symmetric. From our perspective, then, Lorentz covariance emerges as an approximate symmetry of the complete mechanics, rather like isotopic symmetry is an approximate symmetry of nuclear physics. If as we imagine $T$ is of the order of the Planck time or less, then there should be no problem in confronting current particle data, because special relativity has not been tested to anywhere near Planck scales.

This raises an obvious question; why bother to discretise time if all that it does is to produce amplitudes which virtually duplicate conventional field theory? Our answer is that there are known problems with conventional formulations of field theory based on continuous time, such as divergences in Feynman diagrams, and our study is an exploration of an alternative approach to mechanics which might alleviate some of these problems.

Our first paper, referred to as Principles I [1], introduced basic principles for the temporal discretisation of $C T$ classical and quantum particle mechanics. We have recently found a paper by Khorrami [2], who discussed various similar topics and which 
is substantially in agreement with our results. Our second paper, referred to as Principles II [3], extended and applied our principles to classical field theory, including the Schrödinger equation, the Klein-Gordon equation, Maxwell's equations, gauge invariant electrodynamics and the classical Dirac field. The third paper, Principles III, [⿴囗十 tackled scalar quantum field theory and there we discussed the construction of Feynman rules for $D T$ scalar field theory. In that paper we found that otherwise hard or point-like vertices in $C T \varphi^{3}$ Feynman diagrams are replaced by softened vertices in $D T \varphi^{3}$ theory, and that there is a conserved quantity analogous to energy in particle scattering processes, even though there is not a Hamiltonian in the theory. These papers should be consulted for further explanation of our notation, methodology, and motivation. In the present paper we apply the techniques of the previous papers to a discussion of the $D T$ formulation of the quantised Dirac equation. We shall use natural units throughout, where $c=\hbar=1$.

The next section is a review of the fundamentally important $D T$ harmonic oscillator, which provides the basic template for all our field theory propagators and generates the momentum space cutoff predicted in the free particle spectrum. Then we turn to the Dirac equation in one time and zero space dimensions. This provides a toy model which allows us to explore the novelties of $D T$ field theory. This model generates the normal spectrum of particle and anti-particle states, and also entirely novel solutions which, by virtue of their temporal behaviour, we call oscillons and anti-oscillons. The fundamentally bizarre property of oscillons is that they oscillate in phase with a period twice that of the fundamental time $T$. We may readily appreciate that, in the temporal limit $T \rightarrow 0$, these oscillon solutions cannot be accommodated within conventional $C T$ mechanics. If $T$ is extremely small but not zero, we may think of these oscillons as ghost like particles, capable of carrying momentum, spin and charge, but not really a normal form of matter. It is an important result that our quantisation procedure, based on the DT Schwinger action principle, tells us that such single particle oscillon states, were they to be created, would be represented by state vectors having a negative inner product amongst themselves. These states would be regarded as unphysical, according to the usual interpretation of such vectors in state space.

We then turn to the DT Dirac equation in one time and three spatial dimensions. The results are much as with the $1+0$ toy model discussed previously, but with some obvious differences. Now we can consider linear momentum and spin. We show how to construct the corresponding conserved variables using the Maeda-Noether theorem discussed in Principles $I$ and $I I$. We note that in DT we can examine field commutators and anti-commutators in fine detail, and there are one or two surprises with the Dirac field anti-commutators, related to the existence of oscillon solutions. We show also how to couple the DT Dirac equation to the DT Maxwell potentials in a gauge invariant way, and discuss the equations of motion and the conserved electric charge. Finally, we finish with a statement of the DT reduction formulae for the particle and anti-particle in and out states, in preparation for application to $D T$ quantum electrodynamics, to be studied in subsequent papers of this series. 


\section{The Scalar field in $1+0$ dimensions}

In this section we review the inhomogeneous $D T$ harmonic oscillator equation, which serves as the basis for all DT free field equations, including the Klein-Gordon equation, the Dirac equation, and Maxwell's equations.

We showed in Principles $I$ that the classical DT equation of motion for an harmonic oscillator in the presence of a source $j$ is given by the Cadzow equation [1, 5]

$$
\beta x_{n+1}-2 \alpha x_{n}+\beta x_{n-1}=T j_{n}, \quad \beta \neq 0,
$$

where we use the symbol $\underset{c}{=}$ to denote any equality holding on a true or classical trajectory, and $\alpha$ and $\beta$ are constants which are determined by the $C T$ Lagrangian from which the $D T$ system function is constructed. This equation can be written in the form

$$
\left(U_{n}-2 \eta+U_{n}^{-1}\right) x_{n}=J_{c},
$$

where $\eta \equiv \alpha / \beta, J_{n} \equiv T j_{n} / \beta$ and $U_{n}$ is the classical step operator with the property $U_{n} f_{n}=f_{n+1}$ for any variable $f$ indexed by integer $n$. Equation (2) is a second order difference equation. We recall from our previous papers that for this particular equation there are two important dynamical regimes, called the elliptic and hyperbolic regimes, corresponding to $\eta^{2}<1$ and $\eta^{2}>1$ respectively. These are separated by the parabolic barrier, $\eta^{2}=1$. Oscillatory behaviour occurs in the elliptic regime, which in field theories corresponds to the region where physical particle states occur. Singularities in propagators occur at the parabolic barrier in DT field theory, and avoiding these requires some careful discussion.

To solve the equation of motion (1), first define the weighted differences

$$
\Delta x_{n} \equiv x_{n}-\mu x_{n-1},
$$

and suppose that they satisfy the first-order difference equation

$$
\Delta x_{n+1} \underset{c}{=} \lambda \Delta x_{n}+J_{n}
$$

for each value of $n$ considered and for some complex-valued constants $\lambda$ and $\mu$. Then we find

$$
\lambda+\mu=2 \eta, \quad \lambda \mu=1,
$$

which have solutions

$$
\mu=\eta \pm \sqrt{\eta^{2}-1}, \quad \lambda=\eta \mp \sqrt{\eta^{2}-1} .
$$

In the case of forwards propagation, we find

$$
\begin{aligned}
x_{N}=P_{c}^{N-n} & x_{n}-P^{N-n-1} x_{n-1}+P^{N-n-1} J_{n} \\
& +\ldots+P^{1} J_{N-2}+P^{0} J_{N-1}, \quad N>n,
\end{aligned}
$$

whereas for backwards propagation we find

$$
\begin{array}{r}
x_{M}=-P_{c}^{n-M-1} x_{n+1}+P^{n-M} x_{n}+P^{n-M-1} J_{n} \\
+\ldots+P^{1} J_{M+2}+P^{0} J_{M+1}, \quad M<n,
\end{array}
$$


where the polynomials $P^{n} \equiv P^{n}(\lambda, \mu)$ are defined by

$$
P^{n}(\lambda, \mu) \equiv \lambda^{n}+\lambda^{n-1} \mu+\ldots+\lambda \mu^{n-1}+\mu^{n},
$$

where $P^{1}(\lambda, \mu) \equiv \lambda+\mu$ and $P^{0}(\lambda, \mu) \equiv 1$. From (7) and (8) we find

$$
\begin{gathered}
P^{n-M-1} x_{N}+P^{N-n-1} x_{M} \underset{c}{=} \quad\left(P^{n-M-1} P^{N-n}+P^{N-n-1} P^{n-M}-P^{1} P^{n-M-1} P^{N-n-1}\right) x_{n} \\
-P^{n-M-1} P^{N-n-1} J_{n}+P^{n-M-1} \sum_{i=n}^{N-1} P^{N-1-i} J_{i} \\
+P^{N-n-1} \sum_{i=n}^{M+1} P^{i-M-1} J_{i}, \quad M<n<N .
\end{gathered}
$$

This is the desired relationship between the particle's position in the past (assumed known at time $M T<n T$ ), the particle's position in the future (assumed known at time $N T>n T$ ), and the particle's position at the present time $n T$. This equation incorporates Feynman (causal) propagation and the effect of the source terms and is an exact result.

The next step is to look at the scattering limit $N \rightarrow \infty, M \rightarrow-\infty$. Potential singularities at the parabolic barrier are avoided by taking the prescription $\eta \rightarrow$ $\eta+i \varepsilon, \varepsilon>0$, which corresponds to the Feynman $m \rightarrow m-i \varepsilon$ prescription in field theory, where $\epsilon$ is infinitesimal and positive. It is convenient to introduce the parameter $\theta$, related to $\eta$ by $\eta=\cos \theta$ and permitting it to take values in the complex $\theta$ plane only on the contour $\theta=u-i v$, where

$$
\begin{aligned}
& 0 \leq u<\pi, \quad v=\varepsilon>0, \quad \text { (the elliptic regime) } \\
& u=\pi, \quad \varepsilon \leq v<\infty \quad \text { (the hyperbolic regime) }
\end{aligned}
$$

By writing $\eta \equiv x+i y$ we see

$$
\left(\frac{x}{\cosh v}\right)^{2}+\left(\frac{y}{\sinh v}\right)^{2}=1
$$

Points in the elliptic regime lie on the upper half of an ellipse in the complex $\eta$ plane enclosing the points $\eta= \pm 1$, which shows that the parabolic barrier is avoided.

Turning to the $P^{n}$ functions, we use the fact that $\lambda \mu=1$ to find

$$
P^{n}(\lambda, \mu)=\frac{\mu^{n+1}-\mu^{-n-1}}{\mu-\mu^{-1}}, \quad \mu^{2} \neq 1 .
$$

Then taking $\mu=e^{i \theta}=e^{i u+v}$ we find

$$
\lim _{n \rightarrow \infty} \frac{P^{n}}{e^{n(i u+v)}}=\frac{e^{i u+v}}{e^{i u+v}-e^{-i u-v}} .
$$

Now taking the scattering limit $N=-M \rightarrow \infty$ in (11) we find

$$
x_{n} \sim x_{n}^{(0)}-T \sum_{m=-\infty}^{\infty} \Delta_{F}^{n-m} j_{m},
$$


where $x_{n}^{(0)}$ satisfies the source free equation and the $D T$ indexed Feynman propagator $\Delta_{F}^{n}$ satisfies the equation

$$
\left(U_{n}-2 \eta+U_{n}^{-1}\right) \Delta_{F}^{n}=-\Gamma \delta_{n}, \quad \Gamma \equiv \beta^{-1},
$$

where $\delta_{n}$ is the DT Kronecker delta defined in (53). Solving (17) we find

$$
\Delta_{F}^{n}=\frac{\Gamma e^{-i|n| \theta}}{2 i \sin \theta}, \quad \theta=u-i v
$$

which holds for the elliptic and hyperbolic regimes when we take into account the range of values the parameter $\theta$ could take.

Turning to particle theory and the Klein-Gordon equation, we saw in [3] that the various constants in the above equations have the following parametrisations:

$$
\Gamma \equiv \beta^{-1}=\frac{6 T}{6+T^{2} E^{2}}, \quad \eta=\frac{6-2 T^{2} E^{2}}{6+T^{2} E^{2}}
$$

where $E \equiv \sqrt{\mathbf{p} \cdot \mathbf{p}+m^{2}}$ is by definition the energy of the particle and $\mathbf{p}$ is its linear momentum. Now we define the transformed propagator

$$
\tilde{\Delta}_{F}(\mathbf{p}, \Theta) \equiv T \sum_{n=-\infty}^{\infty} e^{i n \Theta} \Delta_{F}^{n}(\mathbf{p})
$$

which satisfies the equation

$$
2(\cos \Theta-\eta) \tilde{\Delta}_{F}(\mathbf{p}, \Theta)=-T \Gamma
$$

Thence we find

$$
\tilde{\Delta}_{F}(\mathbf{p}, \Theta)=\frac{-T \Gamma}{2(\cos \Theta-\eta-i \epsilon)},
$$

using the Feynman $+i \varepsilon$ prescription for avoiding the singularities. In the above we have assumed $\Theta$ is real. We may define the propagator for complex values of $\Theta$ by analytic continuation of (22). If now we introduce the variable $p_{0}$ related to $\Theta$ by the rule

$$
\cos \Theta \equiv \frac{6-2 p_{0}^{2} T^{2}}{6+p_{0}^{2} T^{2}}, \quad \operatorname{sign}(\Theta)=\operatorname{sign}\left(p_{0}\right),
$$

then we find

$$
\tilde{\Delta}_{F}(\mathbf{p}, \Theta)=\frac{1}{\left(p_{0}^{2}-\mathbf{p}^{2}-m^{2}+i \epsilon\right)}+\frac{T^{2} p_{0}^{2}}{6\left(p_{0}^{2}-\mathbf{p}^{2}-m^{2}+i \epsilon\right)},
$$

an exact result. From this we see the emergence of Lorentz symmetry as an approximate symmetry of the mechanics. If $p_{0}$ in the above is taken to represent the same thing in $D T$ as it does in $C T$ special relativity, i.e., the zeroth component of a four-vector, then the first term on the right hand side is clearly a Lorentz scalar, corresponding to the standard bosonic propagator of $C T$ field theory. The second term on the right hand side is not Lorentz invariant but is down on the first term 
by a factor proportional to $T^{2}$. If as we expect $T$ represents an extremely small time scale, such as the Planck time or less, then it is clear that the second term in (24) will be so much smaller in its effects than the first in general and so may be neglected in practice. This is why we expect our DT quantum field theory to be extremely well approximated by Lorentz covariant $C T$ field theory in most situations.

We can use the propagator and the DT Schwinger action principle to calculate the ground state expectation of $D T$ time-ordered products of field operators, and hence extract the free field commutation and anticommutation relations. This was done in Paper III for the scalar field and we shall do the same for the Dirac equation in this paper.

\section{The quantised Dirac particle in $1+0$ dimensions}

In this section we discuss the quantised DT Dirac particle in one time and zero spatial dimensions. This serves as the prototype for the Dirac field studied in the next section. We will use the Schwinger source function technique to obtain the ground state functional in the presence of the sources and from that we are able to extract the ground state expectation values of various anticommutators.

\subsection{Classical equations}

We start with the $C T$ theory. Our dynamical variables are $\psi, \psi^{+}$, which have two fermionic (anticommuting) degrees of freedom each:

$$
\psi=\left[\begin{array}{l}
\psi_{1} \\
\psi_{2}
\end{array}\right], \quad \psi^{+}=\left[\begin{array}{ll}
\psi_{1}^{*} & \psi_{2}^{*}
\end{array}\right],
$$

with $\bar{\psi} \equiv \psi^{+} \gamma^{0}$, where

$$
\gamma^{0}=\left[\begin{array}{cc}
1 & 0 \\
0 & -1
\end{array}\right]
$$

Then the $C T$ Lagrangian is

$$
\begin{aligned}
L & =\frac{1}{2} i \bar{\psi} \gamma^{0} \overrightarrow{\partial_{t}} \psi-\frac{1}{2} i \bar{\psi} \overleftarrow{\partial_{t}} \gamma^{0} \psi-m \bar{\psi} \psi \\
& =\frac{1}{2} i \psi^{+} \dot{\psi}-\frac{1}{2} i \dot{\psi}^{+} \psi-m \psi^{+} \gamma^{0} \psi
\end{aligned}
$$

where we assume the mass $m$ is non-zero. Following Principles $I$ and $I I$ we obtain the $D T$ system function $F^{n}$ from the $C T$ Lagrangian by considering the virtual paths

$$
\begin{aligned}
\tilde{\psi}_{n} & =\lambda \psi_{n+1}+\bar{\lambda} \psi_{n}, \\
\tilde{\psi}_{n}^{+} & =\lambda \psi_{n+1}^{+}+\bar{\lambda} \psi_{n}^{+}, \quad \bar{\lambda} \equiv 1-\lambda,
\end{aligned}
$$

and integrating $T L\left(\tilde{\psi}_{n}, \tilde{\psi}_{n}^{+}\right)$from $\lambda=0$ to $\lambda=1$. With this prescription we find the time derivatives turn into differences. The result is the system function

$$
\begin{aligned}
F^{n}= & \frac{1}{2} i\left\{\psi_{n}^{+} \psi_{n+1}-\psi_{n+1}^{+} \psi_{n}\right\} \\
& -\frac{\kappa}{6}\left\{2 \psi_{n+1}^{+} \gamma^{0} \psi_{n+1}+\psi_{n}^{+} \gamma^{0} \psi_{n+1}+\psi_{n+1}^{+} \gamma^{0} \psi_{n}+2 \psi_{n}^{+} \gamma^{0} \psi_{n}\right\},
\end{aligned}
$$


where $\kappa \equiv m T$.

Next we add the Schwinger sources, which are taken as infinitesimal external disturbances to the system. This may be done in a number of ways. Because we will be interested in vacuum expectation values when the sources are switched off, it does not really matter what we choose to add and how. Our choice turns out to be the most convenient. We define the system function $F^{n}[\eta]$ in the presence of external (i.e. non dynamical) fermionic sources $\eta, \bar{\eta}$ to be given by

$$
F^{n}[\eta] \equiv F^{n}+\frac{1}{2} T\left\{\bar{\eta}_{n} \psi_{n}+\bar{\eta}_{n+1} \psi_{n+1}+\bar{\psi}_{n} \eta_{n}+\bar{\psi}_{n+1} \eta_{n+1}\right\}
$$

and then the Cadzow equation of motion

$$
\frac{\partial}{\partial \psi_{n}^{+}}\left\{F^{n}[\eta]+F^{n-1}[\eta]\right\} \underset{c}{=} 0
$$

gives

$$
\frac{i \gamma^{0}}{2 T}\left(\psi_{n+1}-\psi_{n-1}\right)-\frac{m}{6}\left(\psi_{n+1}+4 \psi_{n}+\psi_{n-1}\right) \underset{c}{=}-\eta_{n}
$$

or

$$
\omega^{+} \psi_{n+1}+4 \kappa \psi_{n}+\omega \psi_{n-1}=6 T \eta_{n}
$$

where $\omega \equiv \kappa+3 i \gamma^{0}$, with a similar equation for the conjugate variable $\psi^{+}$.

Now define the non-singular matrix

$$
\hat{\omega} \equiv \frac{\omega}{|\omega|}=e^{i \xi \gamma^{0}}=\cos \xi+i \sin \xi \gamma^{0}=\left[\begin{array}{cc}
e^{i \xi} & 0 \\
0 & e^{-i \xi}
\end{array}\right],
$$

where $|\omega| \equiv \sqrt{9+\kappa^{2}}$ and the phase angle $\xi$ satisfies (44). Then the equation of motion (33) becomes

$$
\left(\hat{\omega}^{-1} U_{n}-2 \eta+\hat{\omega} U_{n}^{-1}\right) \psi_{n}=\frac{6 T}{|\omega|} \eta_{n}
$$

where $\eta \equiv-2 \kappa /|\omega|$. To solve this equation we first simplify the Dirac space dependence by the non-singular transformation $\psi_{n} \equiv \hat{\omega}^{n} \phi_{n}$ and then the equation of motion becomes

$$
\left(U_{n}-2 \eta+U_{n}^{-1}\right) \phi_{n}=\frac{6 T}{c} \hat{\omega}^{-n} \eta_{n} .
$$

Using the results discussed in $\S 2$ we obtain the formal scattering solution

$$
\phi_{n}=\phi_{n}^{(0)}-T \sum_{m=-\infty}^{\infty} \Delta_{F}^{n-m} \hat{\omega}^{-m} \eta_{m}
$$

where $\phi_{n}^{(0)}$ is a solution of the source free equation and the bosonic propagator satisfies equation (17) . This solution incorporates Feynman scattering boundary conditions. Transforming back to the original fields, we find

$$
\psi_{n}=\psi_{n}^{(0)}-T \sum_{m=-\infty}^{\infty} S_{F}^{n-m} \eta_{m}
$$


where

$$
\begin{aligned}
S_{F}^{n} & \equiv \Delta_{F}^{n} \hat{\omega}^{n}=\frac{6}{2 i|\omega| \sin \theta} e^{-i|n| \theta} \hat{\omega}^{n} \\
& =\frac{6 e^{-i|n| \theta}}{2 i|\omega| \sin \theta}\left\{\cos n \xi+i \sin n \xi \gamma^{0}\right\}
\end{aligned}
$$

is the required $D T$ Dirac propagator. It satisfies the equation

$$
\left(\hat{\omega}^{-1} U_{n}-2 \eta+\hat{\omega} U_{n}^{-1}\right) S_{F}^{n}=-\frac{6}{|\omega|} \delta_{n}
$$

which is equivalent to

$$
\left\{\frac{i \gamma^{0}}{2 T}\left(U_{n}-U_{n}^{-1}\right)-\frac{m}{6}\left(U_{n}+4+U_{n}^{-1}\right)\right\} S_{F}^{n}=\frac{\delta_{n}}{T} .
$$

There are two important angles in this theory, given by

$$
\begin{aligned}
& \cos \theta=\frac{-2 \kappa}{\sqrt{9+\kappa^{2}}}, \quad \sin \theta=\frac{\sqrt{9-3 \kappa^{2}}}{\sqrt{9+\kappa^{2}}} \\
& \cos \xi=\frac{\kappa}{\sqrt{9+\kappa^{2}}}, \quad \sin \xi=\frac{3}{\sqrt{9+\kappa^{2}}}
\end{aligned}
$$

where we assume we are in the elliptic regime $9-3 \kappa^{2}>0$. It is particular linear combinations of these angles which characterise the solutions to the DT Dirac equation, as we shall now show. If we define the angles $\delta \equiv \theta-\xi, \sigma \equiv \theta+\xi$ then we find

$$
\begin{aligned}
S_{F}^{0} & =\frac{3}{i \sqrt{9-3 \kappa^{2}}}, \\
S_{F}^{n} & =\frac{3}{i \sqrt{9-3 \kappa^{2}}}\left[\begin{array}{cc}
e^{-i n \delta} & 0 \\
0 & e^{-i n \sigma}
\end{array}\right], \quad n>0 \\
S_{F}^{n} & =\frac{3}{i \sqrt{9-3 \kappa^{2}}}\left[\begin{array}{cc}
e^{i n \sigma} & 0 \\
0 & e^{i n \delta}
\end{array}\right], \quad n<0 .
\end{aligned}
$$

The significance of the angles $\delta$ and $\xi$ is the following. Assuming the fundamental parameter $T$ is vanishingly small, a Taylor expansion about $\kappa=0$ gives

$$
\begin{aligned}
\delta & \equiv \theta-\xi \simeq \kappa+\frac{1}{180} \kappa^{5}+O\left(\kappa^{7}\right), \\
\sigma & \equiv \theta+\xi \simeq \pi+\frac{1}{3} \kappa+O\left(\kappa^{3}\right) .
\end{aligned}
$$

It turns out that the phase $\delta$ is associated with physical particles in the $C T$ limit $T \rightarrow 0$, whereas $\sigma$ is associated with wave-functions which change sign more-or-less once during each fundamental timestep $T$. This highly oscillatory behaviour is quite different to any behaviour encountered in $C T$ mechanics and leads us to coin the term oscillon for solutions to the equations which behave in such a bizarre way. In 
our theory there will be anti-oscillons as well as oscillons, in the same way there are anti-particles as well as particles.

The reason oscillon solutions occur is not hard to understand. Our DT Dirac equation (32) is, unlike the CT Dirac equation, manifestly second-order in nature. Such an equation will normally have two solutions. However, there are twice as many degrees of freedom when we take into account the Dirac space dimensions in the above theory, so we find a total of four solutions to the DT Dirac equation. These correspond to particle, anti-particle, oscillon, and anti-oscillon solutions. In Paper II we discussed the $D T$ Schrödinger equation in exactly analogous terms, except we did not have a Dirac space structure. So in that example we found particle wavefunction and oscillon wavefunction solutions but no anti-particle or anti-oscillon solutions. We argued there that the oscillon wave-function solutions should not be accessible under ordinary conditions. This is supported by the result we discuss below, that quantum oscillon particle states have a negative norm and so are properly regarded as unphysical.

\subsection{Quantisation}

Having found the propagator the next step is to extract information from it about the field operators. We note that unlike $C T$ mechanics, where we are usually able to impose canonical commutation or anticommutation relations directly, there is more of a problem in DT mechanics. This is because the concept of canonical conjugate momentum becomes close to being redundant in our theory. Fortunately, the Schwinger source functional approach provides a powerful way of finding commutation or anticommutation relations which are consistent with the quantum dynamics.

Given a $D T$ action sum operator $\hat{A}^{N M}[\eta]$, then for evolution from state $|\beta, M\rangle$ at time $T M$ to $|\alpha, N\rangle$ at time $T N(N>M)$ in the presence of external sources, our $D T$ Schwinger action principle is just

$$
\delta\langle\alpha, N \mid \beta, M\rangle_{\eta}=i\left\langle\alpha, N\left|\delta \hat{A}^{N M}[\eta]\right| \beta, M\right\rangle_{\eta}, \quad N>M .
$$

Here the action sum operator is given by

$$
\begin{aligned}
\hat{A}^{N M}[\eta] & \equiv \sum_{n=M}^{N-1} \hat{F}^{n}[\eta] \\
& =\sum_{n=M}^{N-1}\left\{\hat{F}^{n}+\frac{1}{2} T\left[\bar{\eta}_{n+1} \hat{\psi}_{n+1}+\bar{\eta}_{n} \hat{\psi}_{n}+\bar{\psi}_{n+1} \eta_{n+1}+\bar{\psi}_{n} \eta_{n}\right]\right\} .
\end{aligned}
$$

Then we find

$$
\begin{aligned}
\frac{-i}{T} \frac{\partial}{\partial \bar{\eta}_{n}}\langle\alpha, N \mid \beta, M\rangle_{\eta} & =\left\langle\alpha, N\left|\hat{\psi}_{n}\right| \beta, M\right\rangle_{\eta}, \quad N>n>M \\
\frac{i}{T} \frac{\partial}{\partial \eta_{n}}\langle\alpha, N \mid \beta, M\rangle_{\eta} & =\left\langle\alpha, N\left|\bar{\psi}_{n}\right| \beta, M\right\rangle_{\eta}, \quad N>n>M \\
\frac{-i}{T} \frac{\partial}{\partial \bar{\eta}_{N}}\langle\alpha, N \mid \beta, M\rangle_{\eta} & =\frac{1}{2}\left\langle\alpha, N\left|\hat{\psi}_{N}\right| \beta, M\right\rangle_{\eta},
\end{aligned}
$$




$$
\begin{aligned}
\frac{i}{T} \frac{\partial}{\partial \eta_{N}}\langle\alpha, N \mid \beta, M\rangle_{\eta} & =\frac{1}{2}\left\langle\alpha, N\left|\bar{\psi}_{N}\right| \beta, M\right\rangle_{\eta}, \\
\frac{-i}{T} \frac{\partial}{\partial \bar{\eta}_{M}}\langle\alpha, N \mid \beta, M\rangle_{\eta} & =\frac{1}{2}\left\langle\alpha, N\left|\hat{\psi}_{M}\right| \beta, M\right\rangle_{\eta}, \\
\frac{i}{T} \frac{\partial}{\partial \eta_{M}}\langle\alpha, N \mid \beta, M\rangle_{\eta} & =\frac{1}{2}\left\langle\alpha, N\left|\bar{\psi}_{M}\right| \beta, M\right\rangle_{\eta} .
\end{aligned}
$$

We may use these results to work out DT ground state time ordered products. For fermionic fields we note for example

$$
\begin{aligned}
\left\langle 0\left|\tilde{T} \hat{\psi}_{n}^{+} \hat{\psi}_{m}\right| 0\right\rangle \equiv & \left\langle 0\left|\hat{\psi}_{n}^{+} \hat{\psi}_{m}\right| 0\right\rangle \Theta_{n-m}+\frac{1}{2}\left\langle 0\left|\left[\hat{\psi}_{n}^{+}, \hat{\psi}_{n}\right]\right| 0\right\rangle \delta_{n-m} \\
& -\left\langle 0\left|\hat{\psi}_{m} \hat{\psi}_{n}^{+}\right| 0\right\rangle \Theta_{m-n}
\end{aligned}
$$

where $\tilde{T}$ denotes the $D T$ time ordering operator discussed in [1, 3, 4], and $\Theta_{n}$ and $\delta_{n}$ are the $D T$ step and $\delta$ functions respectively, with the properties

$$
\begin{aligned}
\Theta_{n} & =1, \quad n>0, & \delta_{n}=1, & n=0 \\
& =0, \quad n<1, & & =0,
\end{aligned}
$$

We note that in $D T$ we can explicitly discuss what happens when the fields are at equal times, whereas in $C T$ field theory, we tend to avoid this in discussions of time-ordered products, appealing to the properties of distributions.

Now considering the quantum operator equation of motion

$$
\hat{\omega}^{-1} \hat{\psi}_{n+1}+\frac{4 \kappa}{|\omega|} \hat{\psi}_{n}+\hat{\omega} \hat{\psi}_{n-1}=\frac{6 T}{|\omega|} \eta_{n}
$$

we take its matrix element between the ground state, use the above relations (49-51) and hence find the ground state functional

$$
Z[\eta] \equiv\langle 0 \mid 0\rangle_{\eta}=\exp \left\{-i T^{2} \sum_{r, s=-\infty}^{\infty} \bar{\eta}_{r} S_{F}^{r-s} \eta_{s}\right\}
$$

suppressing Dirac space indices. Then using the result

$$
\left\langle 0\left|\tilde{T} \bar{\psi}_{n a} \hat{\psi}_{m b}\right| 0\right\rangle=\lim _{\eta \rightarrow 0}\left\{\frac{i}{T} \frac{\partial}{\partial \eta_{n a}} \frac{-i}{T} \frac{\partial}{\partial \bar{\eta}_{m b}} Z[\eta]\right\}
$$

explicitly showing the Dirac space indices, we find

$$
\left\langle 0\left|\tilde{T} \bar{\psi}_{n a} \hat{\psi}_{m b}\right| 0\right\rangle=-i S_{F b a}^{m-n}
$$

or

$$
\left\langle 0\left|\tilde{T} \hat{\psi}_{n a}^{+} \hat{\psi}_{m b}\right| 0\right\rangle=-i\left(S_{F}^{m-n} \gamma^{0}\right)_{b a}
$$


Using our results for the $D T$ Dirac propagator in the previous subsection and choosing appropriate temporal indices $m$ and $n$, we extract the following information:

$$
\begin{aligned}
n>m:\left\langle 0\left|\psi_{n}^{+} \psi_{m}\right| 0\right\rangle & =-\frac{3}{\sqrt{9-3 \kappa^{2}}} e^{-i(n-m) \theta} \hat{\omega}^{m-n} \gamma^{0} \\
& =\frac{3}{\sqrt{9-3 \kappa^{2}}}\left[\begin{array}{cc}
-e^{-i(n-m) \sigma} & 0 \\
0 & e^{-i(n-m) \delta}
\end{array}\right] \\
\left\langle 0\left|\psi_{m}^{+} \psi_{n}\right| 0\right\rangle & =-\frac{3}{\sqrt{9-3 \kappa^{2}}} \gamma^{0} e^{i(n-m) \theta} \hat{\omega}^{n-m} \\
& =\frac{3}{\sqrt{9-3 \kappa^{2}}}\left[\begin{array}{cc}
-e^{i(n-m) \sigma} & 0 \\
0 & e^{i(n-m) \delta}
\end{array}\right] \\
n\left\langle m:\left\langle 0\left|\psi_{m} \psi_{n}^{+}\right| 0\right\rangle\right. & =\frac{3}{\sqrt{9-3 \kappa^{2}}} e^{-i(m-n) \theta} \hat{\omega}^{m-n} \gamma^{0} \\
& =\frac{3}{\sqrt{9-3 \kappa^{2}}}\left[\begin{array}{cc}
e^{i(n-m) \delta} & 0 \\
0 & -e^{i(n-m) \sigma}
\end{array}\right] \\
\left\langle 0\left|\psi_{n}^{+} \psi_{m}\right| 0\right\rangle & =\frac{3}{\sqrt{9-3 \kappa^{2}}} e^{i(m-n) \theta} \gamma^{0} \hat{\omega}^{n-m} \\
& =\frac{3}{\sqrt{9-3 \kappa^{2}}}\left[\begin{array}{cc}
e^{-i(n-m) \delta} & 0 \\
0 & -e^{-i(n-m) \sigma}
\end{array}\right] .
\end{aligned}
$$

In addition, by taking $n=m$ we find

$$
\left\langle 0\left|\left[\hat{\psi}_{n}, \hat{\psi}_{n}^{+}\right]\right| 0\right\rangle=\frac{3 \gamma^{0}}{2 \sqrt{9-3 \kappa^{2}}} .
$$

By using the $D T$ operator equation of motion for the source free case

$$
\hat{\omega}^{-1} \hat{\psi}_{n+1}+\frac{4 \kappa}{|\omega|} \hat{\psi}_{n}+\hat{\omega} \hat{\psi}_{n-1}=0
$$

we deduce

$$
\left\langle 0\left|\hat{\psi}_{n} \hat{\psi}_{n}^{+}\right| 0\right\rangle=-\left\langle 0\left|\hat{\psi}_{n}^{+} \hat{\psi}_{n}\right| 0\right\rangle=\frac{3 \gamma^{0}}{\sqrt{9-3 \kappa^{2}}} .
$$

We now use the above ground state expectation values and the reasonable assumption that free field anticommutators are c-numbers to write down the following anticommutators:

$$
\begin{aligned}
\left\{\hat{\psi}_{n+1}^{+}, \hat{\psi}_{n}\right\} & =\frac{6 i \gamma^{0}}{|\omega|} \hat{\omega}^{-1}, \\
\left\{\hat{\psi}_{n}^{+}, \hat{\psi}_{n+1}\right\} & =\frac{-6 i \gamma^{0}}{|\omega|} \hat{\omega} \\
\left\{\hat{\psi}_{n}^{+}, \hat{\psi}_{n}\right\} & =0
\end{aligned}
$$

knowing they are consistent with the operator equations of motion and with the propagator. This amounts to our quantisation prescription. 
We note that the last result, (68) is surprising considering that the canonical $C T$ equal-time anticommutator of Dirac field operators is non-zero, i.e.

$$
\left\{\hat{\psi}(t)_{a}, \hat{\psi}^{+}(t)_{b}\right\}=\delta_{a b}
$$

There are two points to be made here:

1. The Schwinger method in $C T$ field theory does not lead to equal time anticommutators directly. It deals with time-ordered products, which involve the Heaviside distribution (the step function) and then we have to consider temporal limits carefully. So for example the $C T$ analogues of the results (59-62) are

$$
\begin{aligned}
& t>0: \quad\left\langle 0\left|\hat{\psi}_{a}^{+}(t) \hat{\psi}_{b}(0)\right| 0\right\rangle=\left[\begin{array}{cc}
0 & 0 \\
0 & e^{-i m t}
\end{array}\right]_{b a} \\
&\left\langle 0\left|\hat{\psi}_{b}^{+}(0) \hat{\psi}_{a}(t)\right| 0\right\rangle=\left[\begin{array}{cc}
0 & 0 \\
0 & e^{i m t}
\end{array}\right]_{b a} \\
& t<0: \quad\left\langle 0\left|\hat{\psi}_{b}(0) \hat{\psi}_{a}^{+}(t)\right| 0\right\rangle=\left[\begin{array}{cc}
e^{i m t} & 0 \\
0 & 0
\end{array}\right]_{b a} \\
&\left\langle 0\left|\hat{\psi}_{a}(t) \hat{\psi}_{b}^{+}(0)\right| 0\right\rangle=\left[\begin{array}{cc}
e^{-i m t} & 0 \\
0 & 0
\end{array}\right]_{b a},
\end{aligned}
$$

from which we deduce

$$
\lim _{t \rightarrow 0^{+}}\left\{\left\langle 0\left|\hat{\psi}_{a}^{+}(t) \hat{\psi}_{b}(0)\right| 0\right\rangle+\left\langle 0\left|\hat{\psi}_{b}(0) \hat{\psi}_{a}^{+}(t)\right| 0\right\rangle\right\}=\delta_{a b}
$$

which is consistent with (69);

2. The value zero in (68) is explained by the presence of oscillon solutions, which also manifest themselves in the relations $(59-62)$. This suggests that the $D T$ field operator $\hat{\psi}_{n}$ is more complicated than its $C T$ analogue $\hat{\psi}(t)$.

\subsection{The free particle Dirac equation}

We turn now to solutions to the free particle DT Dirac equation

$$
\hat{\omega}^{-1} \psi_{n+1}+\frac{4 \kappa}{|\omega|} \psi_{n}+\hat{\omega} \psi_{n} \underset{c}{=} 0
$$

If we write $\psi_{n}=\hat{\omega}^{n} \phi_{n}$ then

$$
\left(U_{n}-2 \eta+U_{n}^{-1}\right) \phi_{n}=0, \quad \eta=\frac{-2 \kappa}{|\omega|} .
$$

Assuming this equation has solutions of the form $\phi_{n}=z^{n} \chi$, where $\chi$ is a two component object and $z$ is taken complex and non-zero, then we find

$$
\left(z-2 \eta+z^{-1}\right) z^{n} \chi_{c}^{=} 0
$$


from which we deduce $z=e^{ \pm i \theta}$, where $\cos \theta=\eta$ satisfies (43). We will assume we are in the elliptic regime, so that $\theta$ is real. Next, define the two component Dirac space basis functions

$$
u \equiv\left[\begin{array}{c}
\sqrt{2 m} \\
0
\end{array}\right], \quad v \equiv\left[\begin{array}{c}
0 \\
\sqrt{2 m}
\end{array}\right]
$$

Then solutions to (74) may be written in the form

$$
\phi_{n}=\frac{1}{2 m}\left\{\left[a e^{-i n \theta}+c^{*} e^{i n \theta}\right] u+\left[d e^{-i n \theta}+b^{*} e^{i n \theta}\right] v\right\} .
$$

We note that there are four independent solutions, as discussed above. Hence finally we get

$$
\begin{aligned}
\psi_{n} & =\hat{\omega}^{n} \phi_{n} \\
& =\frac{1}{2 m}\left\{\left[a e^{-i n \delta}+c^{*} e^{i n \sigma}\right] u+\left[d e^{-i n \sigma}+b^{*} e^{i n \delta}\right] v\right\} .
\end{aligned}
$$

Second quantisation turns the coefficients $a, b, c, d$, etc. into operators, so that the solutions to the quantised DT Dirac equation are

$$
\begin{aligned}
\hat{\psi}_{n} & =\frac{1}{2 m}\left\{\left[\hat{a} e^{-i n \delta}+\hat{c}^{+} e^{i n \sigma}\right] u+\left[\hat{d} e^{-i n \sigma}+\hat{b}^{+} e^{i n \delta}\right] v\right\}, \\
\hat{\psi}_{n}^{+} & =\frac{1}{2 m}\left\{u^{+}\left[\hat{a}^{+} e^{i n \delta}+\hat{c} e^{-i n \sigma}\right]+v^{+}\left[\hat{d}^{+} e^{i n \sigma}+\hat{b} e^{-i n \delta}\right]\right\} .
\end{aligned}
$$

We may invert the relationship (assuming we are in the elliptic regime) to find particleantiparticle creation and annihilation operators:

$$
\begin{aligned}
\hat{a} & =\frac{i}{2 \sin \theta} u^{+}\left\{\hat{\psi}_{n+1} e^{-i \xi}-\hat{\psi}_{n} e^{i \theta}\right\} e^{i n \delta}, & & \rightarrow u^{+} \hat{\psi}(t) e^{i m t} \\
\hat{a}^{+} & =\frac{-i}{2 \sin \theta}\left\{\hat{\psi}_{n+1}^{+} e^{i \xi}-\hat{\psi}_{n}^{+} e^{-i \theta}\right\} u e^{-i n \delta}, & & \rightarrow \hat{\psi}^{+}(t) u e^{-i m t} \\
\hat{b} & =\frac{i}{2 \sin \theta}\left\{\hat{\psi}_{n+1}^{+} e^{-i \xi}-\hat{\psi}_{n}^{+} e^{i \theta}\right\} v e^{i n \delta}, & & \rightarrow \hat{\psi}^{+}(t) v e^{i m t} \\
\hat{b}^{+} & =\frac{-i}{2 \sin \theta} v^{+}\left\{\hat{\psi}_{n+1} e^{i \xi}-\hat{\psi}_{n} e^{-i \theta}\right\} e^{-i n \delta}, & & \underset{T}{\rightarrow} v^{+} \hat{\psi}(t) e^{-i m t}
\end{aligned}
$$

and oscillon/anti-oscillon ladder operators:

$$
\begin{aligned}
\hat{c} & =\frac{i}{2 \sin \theta}\left\{\hat{\psi}_{n+1}^{+} e^{i \xi}-\hat{\psi}_{n}^{+} e^{i \theta}\right\} u e^{i n \sigma}, \quad \vec{T}_{T}^{\rightarrow} 0 \\
\hat{c}^{+} & =\frac{-i}{2 \sin \theta} u^{+}\left\{\hat{\psi}_{n+1} e^{-i \xi}-\hat{\psi}_{n} e^{-i \theta}\right\} e^{-i n \sigma}, \underset{T}{\rightarrow} 0 \\
\hat{d} & =\frac{i}{2 \sin \theta} v^{+}\left\{\hat{\psi}_{n+1} e^{i \xi}-\hat{\psi}_{n} e^{i \theta}\right\} e^{i n \sigma}, \quad \underset{T}{\rightarrow} 0 \\
\hat{d}^{+} & =\frac{-i}{2 \sin \theta}\left\{\hat{\psi}_{n+1}^{+} e^{-i \xi}-\hat{\psi}_{n}^{+} e^{-i \theta}\right\} v e^{-i n \sigma}, \underset{T}{\rightarrow} 0 .
\end{aligned}
$$

In the above equations we show what should happen in the limit

$$
\underset{T}{\rightarrow} \equiv \lim \{T \rightarrow 0, n \rightarrow \infty, n \theta \rightarrow t\}
$$


assuming for example

$$
\psi_{n+1} \simeq \psi(t)+T \dot{\psi}(t)+O\left(T^{2}\right), \quad \text { etc }
$$

We note that we have not indexed the ladder operators with a temporal index $n$, but it is implied. These operators have the same temporal properties as the creation and annihilation operators found for the DT bosonic oscillator discussed in Principles $I$. Although such operators satisfy relations such as $\hat{a}_{n}=\hat{a}_{n+1}$ for example, they are not actually invariants of the motion in that they do not commute with the timestep operator $\hat{U}_{n}$. In the terminology of Principles $I$, these operators are not compatible with the timestep operator. The resolution of this apparent paradox is to note that we are actually working in the Heisenberg picture.

To understand further the basic properties of these operators, we consider the sixty four possible ground state expectation values of products of the eight operators $\hat{a}, \hat{a}^{+}, \ldots, \hat{d}^{+}$. Of these, only four are non-zero. We find

$$
\begin{aligned}
\left\langle 0\left|\hat{a} \hat{a}^{+}\right| 0\right\rangle & =\left\langle 0\left|\hat{b} \hat{b}^{+}\right| 0\right\rangle=\frac{6 m}{\sqrt{9-3 \kappa^{2}}} \\
\left\langle 0\left|\hat{c} \hat{c}^{+}\right| 0\right\rangle & =\left\langle 0\left|\hat{d} \hat{d}^{+}\right| 0\right\rangle=\frac{-6 m}{\sqrt{9-3 \kappa^{2}}} .
\end{aligned}
$$

This is consistent with the view that the operators $\hat{a}, \hat{b}, \hat{c}$, and $\hat{d}$ annihilate the ground state ket $|0\rangle$ and that their adjoints annihilate the ground state bra $\langle 0|$.

If now we use the anticommutation relations $(66-68)$ for the fields worked out previously, we find the following non-zero anticommutators:

$$
\begin{aligned}
& \left\{\hat{a}, \hat{a}^{+}\right\}=\left\{\hat{b}, \hat{b}^{+}\right\}=\frac{6 m}{\sqrt{9-3 \kappa^{2}}}, \underset{T}{\rightarrow} 2 m, \\
& \left\{\hat{c}, \hat{c}^{+}\right\}=\left\{\hat{d}, \hat{d}^{+}\right\}=\frac{-6 m}{\sqrt{9-3 \kappa^{2}}}, \quad \underset{T}{\rightarrow}-2 m .
\end{aligned}
$$

The fundamental result here is that the particle and anti-particle creation and annihilation operators lead to states with a positive inner product, whereas the oscillon and anti-oscillon operators lead to negative norm states, which means that oscillons and anti-oscillons should be regarded as unphysical.

Finally, we find that the conserved charge $\hat{Q}$ operator, given by

$$
\begin{aligned}
\hat{Q}= & \frac{1}{12} \hat{\psi}_{n+1}^{+}\left[3-i \kappa \gamma^{0}\right] \hat{\psi}_{n}+\frac{1}{12} \hat{\psi}_{n}^{+}\left[3+i \kappa \gamma^{0}\right] \hat{\psi}_{n+1} \\
& -\frac{1}{12} \hat{\psi}_{n}\left[3-i \kappa \gamma^{0}\right] \hat{\psi}_{n+1}^{+}-\frac{1}{12} \hat{\psi}_{n+1}\left[3+i \kappa \gamma^{0}\right] \hat{\psi}_{n}^{+}
\end{aligned}
$$

may be written in the form

$$
\hat{Q}=\frac{\sqrt{9-3 \kappa^{2}}}{6 m}\left[\hat{a}^{+} \hat{a}-\hat{b}^{+} \hat{b}+\hat{c}^{+} \hat{c}-\hat{d}^{+} \hat{d}\right] .
$$

Taking the anticommutators into account, we see that particles, antiparticles, oscillons and anti-oscillons carry the same magnitude of charge. We expect therefore that 
whilst oscillons and anti-oscillons would not appear as external in or out asymptotic particles, they can carry charge, linear momentum and spin, and should contribute in Feynman diagrams. In particular, there may be significant novel effects, with particles making virtual transitions to oscillons and back again. When we come to the field theory, discussed next, we expect to find that electric charge as a physical observable should only be associated with particles or antiparticles with momenta below the parabolic barrier.

\section{The Dirac equation in $1+3$ dimensions}

We turn now to the Dirac equation in one time and three spatial dimensions. First we consider the free particle system. Using the virtual paths discussed previously the $C T$ Dirac Lagrangian density

$$
\mathcal{L}(x)=\frac{1}{2} i \bar{\psi}(x) \overrightarrow{\not \partial} \psi(x)-\frac{1}{2} i \bar{\psi}(x) \overleftarrow{\not \partial} \psi(x)-m \bar{\psi}(x) \psi(x)
$$

gives the system function density

$$
\begin{aligned}
\mathcal{F}^{n}(\mathbf{x})= & \frac{1}{2} i\left\{\psi_{n}^{+}(\mathbf{x}) \psi_{n+1}(\mathbf{x})-\psi_{n+1}^{+}(\mathbf{x}) \psi_{n}(\mathbf{x})\right\} \\
& -\frac{T}{6}\left\{\begin{array}{c}
2 \psi_{n+1}^{+}(\mathbf{x}) \overleftrightarrow{H} \psi_{n+1}(\mathbf{x})+\psi_{n}^{+}(\mathbf{x}) \overleftrightarrow{H} \psi_{n+1}(\mathbf{x}) \\
+\psi_{n+1}^{+}(\mathbf{x}) \overleftrightarrow{H} \psi_{n}(\mathbf{x})+2 \psi_{n}^{+}(\mathbf{x}) \overleftrightarrow{H} \psi_{n}(\mathbf{x})
\end{array}\right\}
\end{aligned}
$$

where

$$
\overleftrightarrow{H} \equiv \frac{1}{2} i \overleftarrow{\nabla} \cdot \boldsymbol{\alpha}-\frac{1}{2} i \boldsymbol{\alpha} \cdot \vec{\nabla}+m \beta
$$

is the standard Dirac one-particle Hamiltonian operator. The Cadzow equation for $\psi_{n}(\mathbf{x})$ is formally given by

$$
\frac{\partial}{\partial \psi_{n}^{+}}\left\{\mathcal{F}^{n}+\mathcal{F}^{n-1}\right\} \underset{c}{=} \nabla \cdot \frac{\partial}{\partial \nabla \psi_{n}^{+}}\left\{\mathcal{F}^{n}+\mathcal{F}^{n-1}\right\},
$$

which gives the Cadzow equation for $\psi_{n}$ :

$$
i \frac{\psi_{n+1}-\psi_{n-1}}{2 T}=\frac{1}{6} \vec{H}\left\{\psi_{n+1}+4 \psi_{n}+\psi_{n-1}\right\}
$$

where $\vec{H} \equiv-i \boldsymbol{\alpha} \cdot \vec{\nabla}+m \beta$. In this form we can readily identify the various parts of the non-covariant form of the $C T$ Dirac equation. Another form is

$$
\overrightarrow{\Lambda^{+}} \psi_{n+1}=-\frac{4 i T}{6} \vec{H} \psi_{n}+\vec{\Lambda}^{-} \psi_{n-1}
$$

where $\overrightarrow{\Lambda^{+}} \equiv \frac{1}{2}+\frac{i T}{6} \vec{H}, \overrightarrow{\Lambda^{-}} \equiv \frac{1}{2}-\frac{i T}{6} \vec{H}$. Likewise, we find the Cadzow equation for $\psi_{n}^{+}$

$$
\psi_{n+1}^{+} \overleftarrow{\Lambda^{-}}=\frac{4 i T}{c} \psi_{n}^{+} \overleftarrow{H}+\psi_{n-1}^{+} \overleftarrow{\Lambda^{+}}
$$


where $\overleftarrow{H} \equiv i \overleftarrow{\nabla} \cdot \boldsymbol{\alpha}+m \beta, \overleftarrow{\Lambda^{+}} \equiv \frac{1}{2}+\frac{i T}{6} \overleftarrow{H}$, and $\overleftarrow{\Lambda^{-}} \equiv \frac{1}{2}-\frac{i T}{6} \overleftarrow{H}$. The equations in the form involving the $\Lambda$ operators are useful for proving that various invariants of the motion are indeed constant in time.

With the Fourier transforms

$$
\tilde{\psi}_{n}(\mathbf{p}) \equiv \int d^{3} \mathbf{x} e^{-i \mathbf{p} \cdot \mathbf{x}} \psi_{n}(\mathbf{x}), \quad \tilde{\psi}_{n}^{+}(\mathbf{p}) \equiv \int d^{3} \mathbf{x} e^{i \mathbf{p} \cdot \mathbf{x}} \psi_{n}^{+}(\mathbf{x}),
$$

we find

$$
\begin{aligned}
& \tilde{\Lambda}^{+}(\mathbf{p}) \tilde{\psi}_{n+1}(\mathbf{p})=\frac{-4 i}{c} T H(\mathbf{p}) \tilde{\psi}_{n}(\mathbf{p})+\tilde{\Lambda}^{-}(\mathbf{p}) \tilde{\psi}_{n-1}(\mathbf{p}), \\
& \tilde{\psi}_{n+1}^{+}(\mathbf{p}) \tilde{\Lambda}^{-}(\mathbf{p})=\frac{4 i}{6} T \tilde{\psi}_{n}^{+}(\mathbf{p}) H(\mathbf{p})+\tilde{\psi}_{n-1}^{+}(\mathbf{p}) \tilde{\Lambda}^{+}(\mathbf{p})
\end{aligned}
$$

where $H(\mathbf{p}) \equiv \boldsymbol{\alpha} \cdot \mathbf{p}+m \beta, \tilde{\Lambda}^{+}(\mathbf{p}) \equiv \frac{1}{2}+\frac{i T}{6} H(\mathbf{p})$, and $\tilde{\Lambda}^{-}(\mathbf{p}) \equiv \frac{1}{2}-\frac{i T}{6} H(\mathbf{p})$. Now consider the construction

$$
C^{n} \equiv \int \frac{d^{3} \mathbf{p}}{(2 \pi)^{3}} \mathcal{C}(\mathbf{p})\left\{\tilde{\psi}_{n}(\mathbf{p}) \tilde{\Lambda}^{+}(\mathbf{p}) \tilde{\psi}_{n+1}(\mathbf{p})+\tilde{\psi}_{n+1}(\mathbf{p}) \tilde{\Lambda}^{-}(\mathbf{p}) \tilde{\psi}_{n}(\mathbf{p})\right\}
$$

where $\mathcal{C}(\mathbf{p})$ is arbitrary. Using the equations of motion (98) we readily find

$$
C^{n}=C^{n-1}
$$

which shows that $C^{n}$ is a Logan invariant [1, 6] of the system.

\subsection{The $D T$ Dirac propagator}

In the presence of external fermionic sources the system function density becomes

$$
\mathcal{F}_{\eta}^{n}(\mathbf{x})=\mathcal{F}^{n}(\mathbf{x})+\frac{1}{2} T\left\{\begin{array}{c}
\bar{\eta}_{n}(\mathbf{x}) \psi_{n}(\mathbf{x})+\bar{\eta}_{n+1}(\mathbf{x}) \psi_{n+1}(\mathbf{x}) \\
+\bar{\psi}_{n}(\mathbf{x}) \eta_{n}(\mathbf{x})+\bar{\psi}_{n+1}(\mathbf{x}) \eta_{n+1}(\mathbf{x})
\end{array}\right\}
$$

which gives the Cadzow equation for $\psi_{n}$ :

$$
i \frac{\psi_{n+1}-\psi_{n-1}}{2 T}=\frac{1}{6} \vec{H}\left\{\psi_{n+1}+4 \psi_{n}+\psi_{n-1}\right\}-\beta \eta_{n} .
$$

Likewise, we find the Cadzow equation for $\psi_{n}^{+}$:

$$
\psi_{n+1}^{+} \overleftarrow{\Lambda^{-}}=\frac{4 i T}{c} \psi_{n}^{+} \overleftarrow{H}+\psi_{n-1}^{+} \overleftarrow{\Lambda^{+}}-i T \eta_{n}^{+} \beta
$$

Taking Fourier transforms and with the definitions

$$
\hat{\Omega}(\mathbf{p}) \equiv \frac{\kappa+3 i \hat{H}(\mathbf{p})}{\sqrt{9+\kappa^{2}}}, \quad \kappa \equiv T E_{\mathbf{p}}, \quad \hat{H}(\mathbf{p}) \equiv \frac{H(\mathbf{p})}{E_{\mathbf{p}}}
$$

the equation (102) becomes

$$
\left(\hat{\Omega}^{+}(\mathbf{p}) U_{n}-2 \eta_{E}+\hat{\Omega}(\mathbf{p}) U_{n}^{-1}\right) \tilde{\psi}_{n}(\mathbf{p}) \underset{c}{=} T \Gamma_{E} \hat{H}(\mathbf{p}) \beta \tilde{\eta}_{n}(\mathbf{p})
$$


where

$$
\Gamma_{E} \equiv \frac{6}{\sqrt{9+\kappa^{2}}}, \quad \eta_{E}=\frac{-2 \kappa}{\sqrt{9+\kappa^{2}}} .
$$

With our experience of the $D T$ Dirac equation in $1+0$ dimensions, we may immediately write down the formal solution

$$
\tilde{\psi}_{n}(\mathbf{p})=\tilde{\psi}_{n}^{(0)}(\mathbf{p})-T \sum_{m=-\infty}^{\infty} \tilde{S}_{F}^{n-m}(\mathbf{p}) \tilde{\eta}_{m}(\mathbf{p}),
$$

where $\tilde{\psi}_{n}^{(0)}(\mathbf{p})$ is a solution to the free DT Dirac equation and the propagators

$$
\tilde{S}_{F}^{n}(\mathbf{p}) \equiv \tilde{\Delta}_{F}^{n}(\mathbf{p}) \hat{\Omega}^{n}(\mathbf{p}) \hat{H}(\mathbf{p}) \gamma^{0}, \quad \tilde{\Delta}_{F}^{n}(\mathbf{p}) \equiv \frac{e^{-i|n| \theta_{E}} \Gamma_{E}}{2 i \sin \theta_{E}}
$$

satisfy the equations

$$
\begin{aligned}
\left(\hat{\Omega}^{-1}(\mathbf{p}) U_{n}-2 \eta_{E}+\hat{\Omega}(\mathbf{p}) U_{n}^{-1}\right) \tilde{S}_{F}^{n}(\mathbf{p}) & =-\Gamma_{E} \hat{H}(\mathbf{p}) \beta \delta_{n} \\
\left(U_{n}-2 \eta_{E}+U_{n}^{-1}\right) \tilde{\Delta}_{F}^{n}(\mathbf{p}) & =-\Gamma_{E} \delta_{n} .
\end{aligned}
$$

Note that the bosonic propagator $\tilde{\Delta}_{F}^{n}(\mathbf{p})$ in $(108)$ is dimensionless whereas the bosonic propagator in (18) has the physical dimensions of a length in our system of units.

To investigate the nature of the propagator, define the Fourier series transform

$$
\tilde{S}_{F}(\mathbf{p}, \Theta) \equiv T \sum_{n=-\infty}^{\infty} e^{i n \Theta} \tilde{S}_{F}^{n}(\mathbf{p})
$$

where the parameter $\Theta$ is taken real. Then we find

$$
[\kappa(\cos \Theta+2) \hat{H}-3 \sin \Theta] \tilde{S}_{F}(\mathbf{p}, \Theta)=-3 T \beta .
$$

We may now solve for $\tilde{S}_{F}(\mathbf{p}, \Theta)$ if we give $\kappa^{2}$ a small imaginary term, according to the standard Feynman $m \rightarrow m-i \epsilon$ prescription. Hence we find

$$
\tilde{S}_{F}(\mathbf{p}, \Theta)=\frac{-3 T[\kappa(\cos \Theta+2) \hat{H}-3 \sin \Theta] \beta}{\left[\kappa^{2}(\cos \Theta+2)^{2}-9 \sin ^{2} \Theta-i \epsilon\right]} .
$$

In this form the propagator looks quite different to the standard $C T$ propagator, but a suitable reparametrisation can change this. We introduce the parameter $p_{0}$ (which should not be confused with $E_{p} \equiv \sqrt{\mathbf{p} \cdot \mathbf{p}+m^{2}}$ ) related to the parameter $\Theta$ by

$$
\cos \Theta=\frac{6-2 p_{0}^{2} T^{2}}{6+p_{0}^{2} T^{2}}, \quad \operatorname{sign}(\Theta)=\operatorname{sign}\left(p_{0}\right) .
$$

Then we find

$$
\tilde{S}_{F}(\mathbf{p}, \Theta)=\frac{\gamma^{0} p_{0}+\gamma^{i} p_{i}+m}{p_{0}^{2}-E_{p}^{2}+i \epsilon}+O\left(T^{2}\right)=\frac{\not p+m}{p^{2}-m^{2}+i \epsilon}+O\left(T^{2}\right) .
$$


From this we see that our propagator indeed looks exactly like the $C T$ Feynman propagator for the Dirac field in lowest order in $T$.

This result is important for two reasons. First, the reparametrisation (114) is precisely the same as the parametrisation (23) used in the analogous expansion for the bosonic propagator, (24). This means that the parameter $\Theta$ flowing through Feynman diagram networks has the same representation and interpretation for fermions as it has for bosons, and can be justifiably regarded as the DT analogue of energy, up to a factor of $T$. In Principles III we found that for scalar field theory, the sum of incoming $\Theta$ parameters was conserved at each vertex in a Feynman diagram, and so we conjecture that an analogous result holds for higher spin fields. The second important point is that this results shows us that Lorentz covariance for the Dirac equation emerges from DT mechanics at the same level of approximation as it does in bosonic theory. There is every reason, therefore to regard the loss of manifest Lorentz covariance in the theory as not a serious problem, provided that we work in the regime where $T$ is close to zero in an appropriate sense.

\subsection{Field anticommutators}

With the Fourier series transforms implied by (97) the action sum in the presence of external sources becomes

$$
\begin{aligned}
\hat{A}^{N M}[\eta] \equiv & \sum_{n=M}^{N-1} \int d \mathbf{x} \mathcal{F}^{n}[\eta] \\
= & \hat{A}^{N M}+T \sum_{n=M+1}^{N-1} \int \frac{d \mathbf{p}}{(2 \pi)^{3}}\left[\tilde{\eta}_{n}^{+}(\mathbf{p}) \gamma^{0} \tilde{\psi}_{n}(\mathbf{p})+\tilde{\psi}_{n}^{+}(\mathbf{p}) \gamma^{0} \tilde{\eta}_{n}(\mathbf{p})\right] \\
& +\frac{1}{2} T \int \frac{d \mathbf{p}}{(2 \pi)^{3}}\left\{\tilde{\eta}_{N}^{+}(\mathbf{p}) \gamma^{0} \tilde{\psi}_{N}(\mathbf{p})+\tilde{\psi}_{N}^{+}(\mathbf{p}) \gamma^{0} \tilde{\eta}_{N}(\mathbf{p})\right. \\
& \left.\quad+\tilde{\eta}_{M}^{+}(\mathbf{p}) \gamma^{0} \tilde{\psi}_{M}(\mathbf{p})+\tilde{\psi}_{M}^{+}(\mathbf{p}) \gamma^{0} \tilde{\eta}_{M}(\mathbf{p})\right\} .
\end{aligned}
$$

For the Schwinger action principle, we first define the functional derivatives

$$
\frac{\delta}{\delta \eta_{n}(\mathbf{x})} \eta_{m}(\mathbf{y})=\delta_{n-m} \delta^{3}(\mathbf{x}-\mathbf{y}), \quad \frac{\delta}{\delta \eta_{n}^{+}(\mathbf{x})} \eta_{m}^{+}(\mathbf{y})=\delta_{n-m} \delta^{3}(\mathbf{x}-\mathbf{y})
$$

and

$$
\frac{\delta}{\delta \tilde{\eta}_{n}^{+}(\mathbf{p})} \equiv \int d \mathbf{x} e^{-i \mathbf{p} \cdot \mathbf{x}} \frac{\delta}{\delta \eta_{n}^{+}(\mathbf{x})}, \quad \frac{\delta}{\delta \tilde{\eta}_{n}(\mathbf{p})} \equiv \int d \mathbf{x} e^{i \mathbf{p} \cdot \mathbf{x}} \frac{\delta}{\delta \eta_{n}(\mathbf{x})}
$$

so that

$$
\begin{aligned}
& \frac{\delta}{\delta \tilde{\eta}_{n}(\mathbf{p})} \tilde{\eta}_{m}(\mathbf{q})=(2 \pi)^{3} \delta^{3}(\mathbf{p}-\mathbf{q}) \delta_{n-m} \\
& \frac{\delta}{\delta \tilde{\eta}_{n}^{+}(\mathbf{p})} \tilde{\eta}_{m}^{+}(\mathbf{q})=(2 \pi)^{3} \delta^{3}(\mathbf{p}-\mathbf{q}) \delta_{n-m}
\end{aligned}
$$


Then the Schwinger action principle gives

$$
\begin{aligned}
\frac{-i}{T} \frac{\delta}{\delta \tilde{\eta}_{n}^{+}(\mathbf{p})}\langle\alpha, N \mid \beta, M\rangle_{\eta} & =\left\langle\alpha, N\left|\gamma^{0} \tilde{\psi}_{n}(\mathbf{p})\right| \beta, M\right\rangle_{\eta}, \quad N>n>M \\
\frac{i}{T} \frac{\delta}{\delta \tilde{\eta}_{n}(\mathbf{p})}\langle\alpha, N \mid \beta, M\rangle_{\eta} & =\left\langle\alpha, N\left|\tilde{\psi}_{n}^{+}(\mathbf{p}) \gamma^{0}\right| \beta, M\right\rangle_{\eta}, \quad N>n>M
\end{aligned}
$$

and so on. With this and Cadzow's equations of motion we find the vacuum functional

$$
Z[\eta]=Z[0] \exp \left\{-i T^{2} \sum_{n, m=-\infty}^{\infty} \int \frac{d \mathbf{p}}{(2 \pi)^{3}} \tilde{\eta}_{n}^{+}(\mathbf{p}) \gamma^{0} \tilde{S}_{F}^{n-m}(\mathbf{p}) \tilde{\eta}_{m}(\mathbf{p})\right\},
$$

where

$$
\int \frac{d \mathbf{p}}{(2 \pi)^{3}} e^{i \mathbf{p} \cdot \mathbf{x}} \tilde{S}_{F}^{n}(\mathbf{p})=S_{F}^{n}(\mathbf{x})
$$

We may also write

$$
Z[\eta]=Z[0] \exp \left\{-i T^{2} \sum_{n, m=-\infty}^{\infty} \int d \mathbf{x} d \mathbf{y} \eta_{n}^{+}(\mathbf{x}) \gamma^{0} S_{F}^{n-m}(\mathbf{x}-\mathbf{y}) \eta_{m}(\mathbf{x})\right\} .
$$

where the propagators $S_{F}^{n}(\mathbf{x})$ satisfy the equations

$$
\left\{i \frac{U_{n}^{-1}-U_{n}}{2 T}+\frac{1}{6} \vec{H}\left(U_{n}+4+U_{n}^{-1}\right)\right\} S_{F}^{n}(\mathbf{x})=-\frac{\delta_{n}}{T} \gamma^{0} \delta^{3}(\mathbf{x}) .
$$

Using the rule

$$
\left\langle 0\left|\tilde{T} \tilde{\psi}_{n a}^{+}(\mathbf{p}) \tilde{\psi}_{m b}(\mathbf{q})\right| 0\right\rangle=-i\left[\tilde{S}_{F}^{m-n}(\mathbf{p}) \gamma^{0}\right]_{b a}(2 \pi)^{3} \delta^{3}(\mathbf{p}-\mathbf{q})
$$

we find the following vacuum expectation values:

$$
\begin{aligned}
\left\langle 0\left|\tilde{\psi}_{n+1 a}^{+}(\mathbf{p}) \tilde{\psi}_{n b}(\mathbf{q})\right| 0\right\rangle & =\frac{18 i e^{-i \theta}}{\left(9+\kappa^{2}\right) \sin \theta} \tilde{\Lambda}^{+}(\mathbf{p})_{b a}(2 \pi)^{3} \delta^{3}(\mathbf{p}-\mathbf{q}), \\
\left\langle 0\left|\tilde{\psi}_{n b}^{+}(\mathbf{p}) \tilde{\psi}_{n+1 a}(\mathbf{q})\right| 0\right\rangle & =\frac{-18 i e^{i \theta}}{\left(9+\kappa^{2}\right) \sin \theta} \tilde{\Lambda}^{-}(\mathbf{p})_{a b}(2 \pi)^{3} \delta^{3}(\mathbf{p}-\mathbf{q}), \\
\left\langle 0\left|\tilde{\psi}_{n+1 b}(\mathbf{p}) \tilde{\psi}_{n a}^{+}(\mathbf{q})\right| 0\right\rangle & =\frac{18 i e^{-i \theta}}{\left(9+\kappa^{2}\right) \sin \theta} \tilde{\Lambda}^{-}(\mathbf{p})_{b a}(2 \pi)^{3} \delta^{3}(\mathbf{p}-\mathbf{q}), \\
\left\langle 0\left|\tilde{\psi}_{n a}(\mathbf{p}) \tilde{\psi}_{n+1 b}^{+}(\mathbf{q})\right| 0\right\rangle & =\frac{-18 i e^{i \theta}}{\left(9+\kappa^{2}\right) \sin \theta} \tilde{\Lambda}^{+}(\mathbf{p})_{a b}(2 \pi)^{3} \delta^{3}(\mathbf{p}-\mathbf{q}) .
\end{aligned}
$$

We notice that these are singular at the parabolic barrier. However, by taking anticommutators and we arrive at the fundamental quantisation relations

$$
\begin{aligned}
\left\{\tilde{\psi}_{n+1 a}^{+}(\mathbf{p}), \tilde{\psi}_{n b}(\mathbf{q})\right\} & =\frac{36}{\left(9+\kappa^{2}\right)} \tilde{\Lambda}^{+}(\mathbf{p})_{b a}(2 \pi)^{3} \delta^{3}(\mathbf{p}-\mathbf{q}), \\
\left\{\tilde{\psi}_{n b}^{+}(\mathbf{p}), \tilde{\psi}_{n+1 a}(\mathbf{q})\right\} & =\frac{36}{\left(9+\kappa^{2}\right)} \tilde{\Lambda}^{-}(\mathbf{p})_{a b}(2 \pi)^{3} \delta^{3}(\mathbf{p}-\mathbf{q}), \\
\left\{\tilde{\psi}_{n b}^{+}(\mathbf{p}), \tilde{\psi}_{n a}(\mathbf{q})\right\} & =0,
\end{aligned}
$$

which are, remarkably, free of any singularities at the parabolic barrier. If we had taken commutators instead, we would find that the singularities still occurred at the parabolic barrier. 


\subsection{Ladder operators}

Provided we are in the elliptic regime, the solution to the source free Dirac equation

$$
\left(\hat{\Omega}^{+}(\mathbf{p}) \tilde{\psi}_{n+1}(\mathbf{p})-2 \eta_{E} \tilde{\psi}_{n}(\mathbf{p})+\hat{\Omega}(\mathbf{p}) \tilde{\psi}_{n-1}(\mathbf{p})\right)_{c}^{=0}
$$

is given by

$$
\begin{aligned}
\tilde{\psi}_{n}(\mathbf{p})= & \frac{1}{2 E} \sum_{r=1}^{2}\left\{\left[\hat{a}(\mathbf{p} r) e^{-i n \delta}+\hat{c}(-\mathbf{p} r) e^{i n \sigma}\right] u(\mathbf{p} r)\right. \\
& \left.+\left[\hat{d}^{+}(\mathbf{p} r) e^{-i n \sigma}+\hat{b}^{+}(-\mathbf{p} r) e^{i n \delta}\right] v(-\mathbf{p} r)\right\} .
\end{aligned}
$$

Then as outlined in the $1+0$ case, we find:

for the particles:

$$
\begin{aligned}
\hat{a}(\mathbf{p} r) & =\frac{i}{2 \sin \theta} u^{+}(\mathbf{p} r)\left\{\tilde{\psi}_{n+1}(\mathbf{p}) e^{-i \xi}-\tilde{\psi}_{n}(\mathbf{p}) e^{i \theta}\right\} e^{i n \delta}, \\
\hat{a}^{+}(\mathbf{p} r) & =\frac{-i}{2 \sin \theta}\left\{\tilde{\psi}_{n+1}^{+}(\mathbf{p}) e^{i \xi}-\tilde{\psi}_{n}^{+}(\mathbf{p}) e^{-i \theta}\right\} u(\mathbf{p} r) e^{-i n \delta}, \\
\hat{b}(\mathbf{p} r) & =\frac{i}{2 \sin \theta}\left\{\tilde{\psi}_{n+1}^{+}(-\mathbf{p}) e^{-i \xi}-\tilde{\psi}_{n}^{+}(-\mathbf{p}) e^{i \theta}\right\} v(\mathbf{p} r) e^{i n \delta}, \\
\hat{b}^{+}(\mathbf{p} r) & =\frac{-i}{2 \sin \theta} v^{+}(\mathbf{p} r)\left\{\tilde{\psi}_{n+1}(-\mathbf{p}) e^{i \xi}-\tilde{\psi}_{n}(-\mathbf{p}) e^{-i \theta}\right\} e^{-i n \delta},
\end{aligned}
$$

and for the oscillons:

$$
\begin{aligned}
\hat{c}(\mathbf{p} r) & =\frac{i}{2 \sin \theta}\left\{\tilde{\psi}_{n+1}^{+}(-\mathbf{p}) e^{i \xi}-\tilde{\psi}_{n}^{+}(-\mathbf{p}) e^{i \theta}\right\} u(-\mathbf{p} r) e^{i n \sigma}, \\
\hat{c}^{+}(\mathbf{p} r) & =\frac{-i}{2 \sin \theta} u^{+}(-\mathbf{p} r)\left\{\tilde{\psi}_{n+1}(-\mathbf{p}) e^{-i \xi}-\tilde{\psi}_{n}(-\mathbf{p}) e^{-i \theta}\right\} e^{-i n \sigma}, \\
\hat{d}(\mathbf{p} r) & =\frac{i}{2 \sin \theta} v^{+}(-\mathbf{p} r)\left\{\tilde{\psi}_{n+1}(\mathbf{p}) e^{i \xi}-\tilde{\psi}_{n}(\mathbf{p}) e^{i \theta}\right\} e^{i n \sigma} \\
\hat{d}^{+}(\mathbf{p} r) & =\frac{-i}{2 \sin \theta}\left\{\tilde{\psi}_{n+1}^{+}(\mathbf{p}) e^{-i \xi}-\tilde{\psi}_{n}^{+}(\mathbf{p}) e^{-i \theta}\right\} v(-\mathbf{p} r) e^{-i n \sigma}
\end{aligned}
$$

Here we have used conventional Dirac momentum spinors defined by

$$
\begin{aligned}
& u(\mathbf{p} r) \equiv \frac{(\not p+m)}{\sqrt{m+E}} u_{r}=\frac{1}{\sqrt{m+E}}\left[\begin{array}{c}
(m+E) \chi_{r} \\
\mathbf{p} \cdot \boldsymbol{\sigma} \chi_{r}
\end{array}\right], \\
& v(\mathbf{p} r) \equiv \frac{(m-\not p)}{\sqrt{m+E}} v_{r}=\frac{1}{\sqrt{m+E}}\left[\begin{array}{c}
\mathbf{p} \cdot \boldsymbol{\sigma} \eta_{r} \\
(m+E) \eta_{r}
\end{array}\right]
\end{aligned}
$$

with

$$
\hat{H}(\mathbf{p}) u(\mathbf{p} r)=u(\mathbf{p} r), \quad \hat{H}(-\mathbf{p}) v(\mathbf{p} r)=-v(\mathbf{p} r) .
$$

Now using the field anti-commutators, we arrive at the following non-zero creation and annihilation relations: for the particles we have

$$
\left\{\hat{a}(\mathbf{p} r), \hat{a}^{+}(\mathbf{q} s)\right\}=\left\{\hat{b}(\mathbf{p} r), \hat{b}^{+}(\mathbf{q} s)\right\}=\frac{6 E}{\sqrt{9-3 \kappa^{2}}} \delta_{r s}(2 \pi)^{3} \delta^{3}(\mathbf{p}-\mathbf{q}),
$$


whereas for the oscillons we find

$$
\left\{\hat{c}(\mathbf{p} r), \hat{c}^{+}(\mathbf{q} s)\right\}=\left\{\hat{d}(\mathbf{p} r), \hat{d}^{+}(\mathbf{q} s)\right\}=\frac{-6 E}{\sqrt{9-3 \kappa^{2}}} \delta_{r s}(2 \pi)^{3} \delta^{3}(\mathbf{p}-\mathbf{q}) .
$$

All other anticommutators are zero. It is clear we should be in the elliptic regime for any of these anticommutators to make physical sense. Moreover, we see that even though their linear momenta may be in the elliptic regime $T E_{\mathbf{p}}<\sqrt{3}$, oscillon and anti-oscillon particle states have a negative inner product and are therefore unphysical. This confirms the results of the previous section.

\subsection{Linear Momentum}

When there are continuous symmetries of the system function we may construct various invariants of the motion using the Maeda-Noether theorem discussed in Prin-

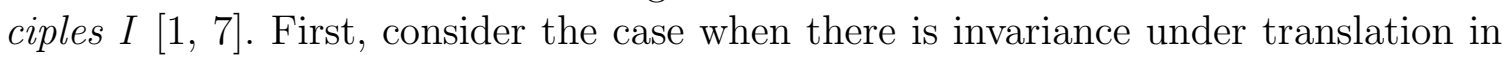
space. From the results of Principles II [3] we construct the three components of the conserved linear momentum using the rule

$$
\begin{aligned}
& \mathbf{P}^{n} \equiv \int d \mathbf{x}\left\{\left[\mathcal{F}^{n} \frac{\overleftarrow{\partial}}{\partial \psi_{n}}\right.\right.\left.-\nabla \cdot\left(\mathcal{F}^{n} \frac{\overleftarrow{\partial}}{\partial \nabla \psi_{n}}\right)\right] \vec{\nabla} \psi_{n} \\
&\left.+\psi_{n}^{+} \overleftarrow{\nabla}\left[\frac{\vec{\partial}}{\partial \psi_{n}^{+}} \mathcal{F}^{n}-\nabla \cdot\left(\frac{\partial}{\partial \nabla \psi_{n}^{+}} \mathcal{F}^{n}\right)\right]\right\} .
\end{aligned}
$$

This takes into account the anticommutation properties of the fields. Applying this rule to the translation invariant source free system function density (91) we find

$$
\mathbf{P}^{n}=i \int d \mathbf{x}\left\{\psi_{n}^{+} \overleftarrow{\nabla} \overrightarrow{\Lambda^{+}} \psi_{n+1}-\psi_{n+1}^{+} \overleftarrow{\Lambda^{-}} \vec{\nabla} \psi_{n}\right\}
$$

which has the appropriate limit

$$
\lim _{T \rightarrow 0} \mathbf{P}^{n}=\int d \mathbf{x}\left\{\frac{1}{2} i \psi^{+} \overleftarrow{\nabla} \psi-\frac{1}{2} i \psi^{+} \vec{\nabla} \psi\right\}
$$

as expected. Using the equations of motion (95, 96) we readily find

$$
\mathbf{P}^{n}=\mathbf{P}^{n+1}
$$

as expected. In the proof of this result we have to integrate by parts and assume that the wave-function falls off to zero at spatial infinity, which is to be expected for physical, i.e. normalisable states.

Taking Fourier transforms, we find

$$
\mathbf{P}^{n}=\int \frac{d \mathbf{p}}{(2 \pi)^{3}} \mathbf{p}\left\{\tilde{\psi}_{n}^{+}(\mathbf{p}) \tilde{\Lambda}^{+}(\mathbf{p}) \tilde{\psi}_{n+1}(\mathbf{p})+\tilde{\psi}_{n+1}^{+}(\mathbf{p}) \tilde{\Lambda}^{-}(\mathbf{p}) \tilde{\psi}_{n}(\mathbf{p})\right\}
$$


which on comparison with (99) shows that the linear momentum is an example of a Logan invariant. After quantisation and using the results (127) we find the commutators

$$
\begin{aligned}
& {\left[\hat{\mathbf{P}}^{n}, \hat{a}^{+}(\mathbf{p} r)\right]=\mathbf{p} \hat{a}^{+}(\mathbf{p} r), \quad\left[\hat{\mathbf{P}}^{n}, \hat{b}^{+}(\mathbf{p} r)\right]=\mathbf{p} \hat{b}^{+}(\mathbf{p} r)} \\
& {\left[\hat{\mathbf{P}}^{n}, \hat{c}^{+}(\mathbf{p} r)\right]=\mathbf{p} \hat{c}^{+}(\mathbf{p} r), \quad\left[\hat{\mathbf{P}}^{n}, \hat{d}^{+}(\mathbf{p} r)\right]=\mathbf{p} \hat{d}^{+}(\mathbf{p} r)}
\end{aligned}
$$

which shows that these operators do indeed create and annihilate excitations carrying definite linear momentum.

\subsection{Angular momentum}

Consider the free Dirac system function (91). An infinitesimal rotation gives the following changes in the fields:

$$
\delta \psi_{n}=i \boldsymbol{\omega} \cdot \overrightarrow{\mathbf{J}} \psi_{n}, \quad \delta \psi_{n}^{+}=-i \psi_{n} \overleftarrow{\mathbf{J}} \cdot \boldsymbol{\omega}
$$

where

$$
\overrightarrow{\mathbf{J}} \equiv-i \mathbf{x} \times \vec{\nabla}+\frac{1}{2} \boldsymbol{\Sigma}, \quad \overleftarrow{\mathbf{J}} \equiv i \mathbf{x} \times \overleftarrow{\nabla}+\frac{1}{2} \boldsymbol{\Sigma}
$$

The system function is invariant to this transformation and so we may use the MaedaNoether theorem to find the conserved angular momentum:

$$
\mathbf{J}_{n}=\int d \mathbf{x}\left\{\psi_{n}^{+} \overleftarrow{\mathbf{J}} \overrightarrow{\Lambda^{+}} \psi_{n+1}+\psi_{n+1}^{+} \overleftarrow{\Lambda^{-}} \overrightarrow{\mathbf{J}} \psi_{n}\right\}
$$

With the Cadzow equations (95, 96) we find

$$
\mathbf{J}_{n}=\mathbf{J}_{n-1}
$$

as expected. We note in passing

$$
[\vec{H}, \overrightarrow{\mathbf{J}}]=\mathbf{0} .
$$

a relation encountered in the $C T$ Dirac equation.

\section{The charged Dirac equation}

We now discuss the coupling of electromagnetic potentials to the Dirac field. We follow here the DT formulation of the Maxwell fields discussed in Principles II [3], treating these fields as external, i.e., non-dynamical, and so in this paper we consider only the dynamics of the Dirac field. A full discussion of QED is reserved for subsequent papers in this series.

In DT gauge invariant electromagnetism, the electrostatic (scalar) potential $\phi$ is associated with the temporal links between successive instants of time, whereas the magnetic (vector) potential $\mathbf{A}$ is associated with the sites themselves. If $\phi_{n}(\mathbf{x})$ 
denotes the scalar potential at position $\mathbf{x}$ on the temporal link $(n, n+1)$ and $\mathbf{A}_{n}(\mathbf{x})$ is associated with the position $\mathbf{x}$ at time $n$, then under a gauge transformation we have

$$
\begin{aligned}
\phi_{n}^{\prime}(\mathbf{x}) & =\phi_{n}(\mathbf{x})+\frac{\chi_{n+1}(\mathbf{x})-\chi_{n}(\mathbf{x})}{T} \\
\mathbf{A}_{n}^{\prime}(\mathbf{x}) & =\mathbf{A}_{n}(\mathbf{x})-\nabla \chi_{n}(\mathbf{x}) \\
\psi_{n}^{\prime}(\mathbf{x}) & =X_{n}(\mathbf{x}) \psi_{n}(\mathbf{x})
\end{aligned}
$$

where $X_{n}(\mathbf{x}) \equiv \exp \left\{-i q \chi_{n}(\mathbf{x})\right\}, q$ being the charge associated with the Dirac field.

Turning to virtual paths, the presence of electromagnetic potentials requires a modification of the free field paths used previously. Following our discussion of the charged Klein-Gordon field in Principles II, we define the paths

$$
\begin{aligned}
\tilde{\psi}_{n} & \equiv \lambda W_{n}^{\bar{\lambda}} \psi_{n+1}+\bar{\lambda} W_{n}^{-\lambda} \psi_{n}, \\
\tilde{\psi}_{n}^{+} & \equiv \lambda W_{n}^{-\bar{\lambda}} \psi_{n+1}^{+}+\bar{\lambda} W_{n}^{\lambda} \psi_{n}^{+},
\end{aligned}
$$

where $W_{n} \equiv \exp \left(i q \phi_{n} T\right)$. The gauge covariant derivatives are given by

$$
\begin{aligned}
\overrightarrow{D_{n}} \tilde{\psi}_{n} & \equiv\left(\frac{\partial_{\lambda}}{T}+i q \phi_{n}\right) \tilde{\psi}_{n}=\frac{W_{n}^{\bar{\lambda}} \psi_{n+1}-W_{n}^{-\lambda} \psi_{n}}{T} \\
\overrightarrow{\mathbf{D}}_{n} \tilde{\psi}_{n} & \equiv\left(\vec{\nabla}-i q \tilde{\mathbf{A}}_{n}\right) \tilde{\psi}_{n} \\
& =\lambda W_{n}^{\bar{\lambda}}\left[i q T \bar{\lambda} \nabla \phi_{n}+\vec{\nabla}_{n}-i q \tilde{\mathbf{A}}_{n}\right] \psi_{n+1}+\bar{\lambda} W_{n}^{-\lambda}\left[-i q T \lambda \nabla \phi_{n}+\vec{\nabla}_{n}-i q \tilde{\mathbf{A}}_{n}\right] \psi_{n},
\end{aligned}
$$

where the virtual paths for the electromagnetic potentials are given by

$$
\tilde{\phi}_{n} \equiv \phi_{n}, \quad \tilde{\mathbf{A}}_{n} \equiv \lambda \mathbf{A}_{n+1}+\bar{\lambda} \mathbf{A}_{n}
$$

Then under a gauge transformation, we find

$$
\begin{aligned}
& \overrightarrow{D_{n}^{\prime}} \tilde{\psi}_{n}^{\prime}=X_{n+1}^{\lambda} X_{n}^{\bar{\lambda}} \vec{D}_{n} \tilde{\psi}_{n}=\tilde{X}_{n} \vec{D}_{n} \tilde{\psi}_{n}, \\
& \overrightarrow{\mathbf{D}_{n}^{\prime}} \tilde{\psi}_{n}^{\prime}=X_{n+1}^{\lambda} X_{n}^{\bar{\lambda}} \overrightarrow{\mathbf{D}_{n}} \tilde{\psi}_{n}=\tilde{X}_{n} \overrightarrow{\mathbf{D}_{n}} \tilde{\psi}_{n} \text {. }
\end{aligned}
$$

The gauge invariant system function for the Dirac field in external electromagnetic potentials may be given in the form

$$
F^{n}=\frac{1}{2} T \int d \mathbf{x}\left\{\left\langle\tilde{\psi}_{n}^{+} \overrightarrow{\mathcal{O}}_{n} \tilde{\psi}_{n}\right\rangle+\left\langle\tilde{\psi}_{n}^{+} \overleftarrow{\mathcal{O}}_{n} \tilde{\psi}_{n}\right\rangle\right\}
$$

where we use the angular brackets to denote integration over $\lambda$, i.e.

$$
\langle f\rangle \equiv \int_{0}^{1} f(\lambda) d \lambda
$$

and

$$
\begin{aligned}
& \overrightarrow{\mathcal{O}_{n}} \equiv i \overrightarrow{D_{n}}+i \boldsymbol{\alpha} \cdot \overrightarrow{\mathbf{D}_{n}}-m \beta \\
& \overleftarrow{\mathcal{O}_{n}} \equiv-i \overleftarrow{D_{n}}-i \boldsymbol{\alpha} \cdot \overleftarrow{\mathbf{D}_{n}}-m \beta .
\end{aligned}
$$


Then we find the Cadzow equations of motion for the Dirac fields are

$$
\begin{aligned}
& \left\langle\bar{\lambda} W_{n}^{\lambda} \overrightarrow{\mathcal{O}_{n}} \tilde{\psi}_{n}\right\rangle+\left\langle\lambda W_{n-1}^{-\bar{\lambda}} \overrightarrow{\mathcal{O}_{n-1}} \tilde{\psi}_{n-1}\right\rangle=0 \\
& \left\langle\tilde{\psi}_{n}^{+} \overleftarrow{\mathcal{O}_{n}} \bar{\lambda} W_{n}^{-\lambda}\right\rangle+\left\langle\tilde{\psi}_{n-1}^{+} \overleftarrow{\mathcal{O}_{n-1}} \lambda W_{n-1}^{\bar{\lambda}}\right\rangle \underset{c}{=} 0 .
\end{aligned}
$$

We note that, as found in Principles III, there is often an advantage in not evaluating the integration over the virtual path until a late stage in a calculation.

To go further, we introduce the following notation

$$
\begin{array}{ll}
A_{n} \equiv \lambda W_{n-1}^{\bar{\lambda}} \psi_{n}, & B_{n} \equiv \bar{\lambda} W_{n}^{-\lambda} \psi_{n} \\
A_{n}^{+} \equiv \lambda W_{n-1}^{-\bar{\lambda}} \psi_{n}^{+}, & B_{n}^{+} \equiv \bar{\lambda} W_{n}^{\lambda} \psi_{n}^{+} .
\end{array}
$$

Then we have

$$
\tilde{\psi}_{n}=A_{n+1}+B_{n}, \quad \tilde{\psi}_{n}^{+}=A_{n+1}^{+}+B_{n}^{+}
$$

and then the Cadzow equations give the relations

$$
\begin{aligned}
& \left\langle B_{n}^{+} \overrightarrow{\mathcal{O}_{n}}\left(A_{n+1}+B_{n}\right)\right\rangle+\left\langle A_{n}^{+} \overrightarrow{\mathcal{O}_{n-1}}\left(A_{n}+B_{n-1}\right)\right\rangle=\underset{c}{=} 0 \\
& \left\langle\left(A_{n+1}^{+}+B_{n}^{+}\right) \overleftarrow{\mathcal{O}_{n}} B_{n}\right\rangle+\left\langle\left(A_{n}^{+}+B_{n-1}^{+}\right) \overleftarrow{\mathcal{O}_{n-1}} A_{n}\right\rangle \underset{c}{=} 0
\end{aligned}
$$

These relations are very useful for proving charge conservation. Under gauge transformations where

$$
W_{n}^{\prime}=X_{n+1}^{-1} W_{n} X_{n}, \quad X_{n} \equiv \exp \left\{-i q \chi_{n}\right\}, \quad \psi_{n}^{\prime}=X_{n} \psi_{n},
$$

then

$$
A_{n}^{\prime}=\tilde{X}_{n-1} A_{n}, \quad B_{n}^{\prime}=\tilde{X}_{n} B_{n}, \quad \tilde{\psi}_{n}^{\prime}=\tilde{X}_{n} \tilde{\psi}_{n}
$$

where

$$
\tilde{X}_{n}=\exp \left\{-i q\left(\lambda \chi_{n+1}+\bar{\lambda} \chi_{n}\right\}=X_{n+1}^{\lambda} X_{n}^{\bar{\lambda}} .\right.
$$

To find the total charge consider the infinitesimal global gauge transformation

$$
\delta \psi_{n}=-i q \delta \chi \psi_{n}, \quad \delta \psi_{n}^{+}=i q \delta \chi \psi_{n}^{+}
$$

and apply the Maeda-Noether theorem discussed in Principles II. Then we find the total charge can be written in the form

$$
Q^{n}=i q T \int d \mathbf{x}\left\{\left\langle A_{n+1}^{+} \overleftarrow{\mathcal{O}_{n}} B_{n}\right\rangle-\left\langle B_{n}^{+} \overrightarrow{\mathcal{O}_{n}} A_{n+1}\right\rangle\right\}
$$

Then modulo the equations of motion (161) we readily find

$$
Q^{n}=Q^{n-1}
$$

and

$$
\lim _{T \rightarrow 0} Q^{n}=q \int d \mathbf{x} \psi^{+} \psi
$$

as expected. 


\subsection{Reduction formulae}

We anticipate now the construction of scattering amplitudes to be discussed in detail in subsequent papers of this series by giving the reduction formulae involving the Dirac particle and anti-particle creation and annihilation operators. If $\tilde{T}$ denotes the discrete time ordering operator, discussed above and $\overrightarrow{U_{n}}$ is the classical step function defined previously then we find the following reduced matrix elements:

$$
\begin{aligned}
\left\langle\alpha_{\text {out }}\left|a_{\text {out }}(\mathbf{p} r)(\tilde{T} \hat{O})\right| \beta_{\text {in }}\right\rangle_{R} & =\frac{i}{2 \sin \theta} \sum_{n=-\infty}^{\infty} e^{i n \delta} u^{+}(\mathbf{p} r) \overrightarrow{\mathcal{D}(\mathbf{p})}\left\langle\alpha_{\text {out }}\left|\tilde{T}\left(\psi_{n}(\mathbf{p}) \hat{O}\right)\right| \beta_{\text {in }}\right\rangle, \\
\left\langle\alpha_{\text {out }}\left|(\tilde{T} \hat{O}) a_{\text {in }}^{+}(\mathbf{p} r)\right| \beta_{\text {in }}\right\rangle_{R} & =\frac{i}{2 \sin \theta} \sum_{n=-\infty}^{\infty}\left\langle\alpha_{\text {out }}\left|\tilde{T}\left(\hat{O} \psi_{n}^{+}(\mathbf{p})\right)\right| \beta_{\text {in }}\right\rangle \overleftarrow{\mathcal{D}(\mathbf{p})} u(\mathbf{p} r) e^{-i n \delta}, \\
\left\langle\alpha_{\text {out }}\left|b_{\text {out }}(\mathbf{p} r)(\tilde{T} \hat{O})\right| \beta_{\text {in }}\right\rangle_{R} & =\frac{i}{2 \sin \theta} \sum_{n=-\infty}^{\infty}\left\langle\alpha_{\text {out }}\left|\tilde{T}\left(\psi_{n}^{+}(-\mathbf{p}) \hat{O}\right)\right| \beta_{\text {in }}\right\rangle \overleftarrow{\mathcal{D}(-\mathbf{p})} v(\mathbf{p} r) e^{\text {in } \delta}, \\
\left\langle\alpha_{\text {out }}\left|(\tilde{T} \hat{O}) b_{\text {in }}^{+}(\mathbf{p} r)\right| \beta_{\text {in }}\right\rangle_{R} & =\frac{i}{2 \sin \theta} \sum_{n=-\infty}^{\infty} e^{-i n \delta} v^{+}(\mathbf{p} r) \overrightarrow{\mathcal{D}(-\mathbf{p})}\left\langle\alpha_{\text {out }}\left|\tilde{T}\left(\hat{O} \psi_{n}(-\mathbf{p})\right)\right| \beta_{\text {in }}\right\rangle
\end{aligned}
$$

where

$$
\begin{aligned}
& \overrightarrow{\mathcal{D}(\mathbf{p})} \equiv \hat{\Omega}^{+}(\mathbf{p}) \overrightarrow{U_{n}}-2 \eta_{\mathbf{p}}+\hat{\Omega}(\mathbf{p}) \overrightarrow{U_{n}^{-1}} \\
& \overleftarrow{\mathcal{D}(\mathbf{p})} \equiv \overleftarrow{U_{n}} \hat{\Omega}(\mathbf{p})-2 \eta_{\mathbf{p}}+\overleftarrow{U_{n}^{-1}} \hat{\Omega}^{+}(\mathbf{p})
\end{aligned}
$$

In these matrix elements we have discarded the non-scattered components, as usual. In addition, we have not given any reduction formulae for the oscillon and anti-oscillon ladder operators. These we regard as not creating physically accessible states, and therefore, they should not be put on the same footing as the particle and anti-particle creation and annihilation operators.

\section{Concluding remarks}

The introduction of a non-zero $T$ opens up a crack in $C T$ quantum field theory which admits a number of new phenomena. In addition to the novelties encountered previously in DT bosonic theories, such as a physical particle momentum cutoff, modified propagators and vertices, we find in the case of the Dirac equation the appearance of fermionic oscillon and anti-oscillon solutions. These should not survive in the $C T$ limit on account of their extraordinary properties. For non-zero $T$ they should certainly participate in DT Feynman diagram processes as intermediate, virtual objects carrying charge, linear momentum and spin, but we expect them not to be observable as asymptotic in or out particles. We plan to investigate their role in DT QED scattering processes and regularisation in subsequent papers in this series. 


\section{Acknowledgement}

Keith Norton is grateful to the Crowther Fund of the Open University for financial assistance during this course of this research.

\section{References}

[1] Jaroszkiewicz G and Norton K, Principles of Discrete Time Mechanics: I. Particle Systems, 3115-3144, J. Phys. A: Math. Gen. 30, No 9, 7 May 1997

[2] Khorrami, M, A general formulation of discrete-time quantum mechanics, restrictions on the action and the relation of unitarity to the existence theorem for initial-value problems, http://xxx.lanl.gov/quant-ph/9412008, Annals Phys. 244 (1995) 101-111.

[3] Jaroszkiewicz G and Norton K, Principles of Discrete Time Mechanics: II. Classical Field Theory, 3145-3163, J. Phys. A: Math. Gen. 30, No 9, 7 May 1997

[4] Norton K and Jaroszkiewicz G, Principles of Discrete Time Mechanics: III. Quantum Field Theory, 977-1000, J.Phys.A: Math. Gen. 31, No 3, 23 January 1998

[5] Cadzow J A, Discrete Calculus of Variations, Int. J. Control, vol 11, No 3, 393-407 (1970)

[6] Logan J D, First Integrals in the Discrete Variational Calculus, Aequat. Math. $\mathbf{9}, 210-220(1973)$

[7] Maeda S, Extension of Discrete Noether Theorem, Math. Japonica 26, no 1, 85-90 (1981) and references therein. 